\title{
Neocollagenesis in Non-Invasive Aesthetic Treatments
}

\author{
Ilja L. Kruglikov* \\ Wellcomet GmbH, Karlsruhe, Germany. \\ Email: i.kruglikov@wellcomet.de \\ Received October $8^{\text {th }}, 2012$; revised November $10^{\text {th }}, 2012$; accepted November $19^{\text {th }}, 2012$
}

\begin{abstract}
Dermal neocollagenesis is often assumed to be the main reason of visible skin improvement after different non-invasive and minimal invasive aesthetic treatments. However, the very slow dynamics of the mature collagen remodelling in the extra cellular matrix (ECM) of dermis, with a half-life time of 15 years, renders every observable upregulation of collagen production insufficient to replace a significant part of the matrix during the short time in which it is claimed skin improvement takes place.
\end{abstract}

Keywords: Neocollagenesis; Aesthetic Treatments; Procollagen; Mature Collagen; Turnover

\section{Introduction}

Skin rejuvenation using ablative and non-ablative procedures based on different light sources (laser, IPL, LED), radio-frequency (monopolar, bi-polar, fractional, etc.), ultrasound as well as combinations of these have become popular in aesthetic medicine. Almost all treatment modalities based on these physical methods claim the main effect of their application to be the thermic or athermic stimulation of neocollagenesis. This inspires the feeling that neocollagenesis is a non-specific process, which can be stimulated through the application of almost every physical force, even at very low intensities. Modulation of the dermal ECM as the main goal of non-invasive or minimally-invasive aesthetic treatment, especially the stimulation of the collagen production de novo, is not an accidental choice. The ECM, with its insoluble scaffoldconsisting mainly of stiff, mature fibrillar collagenprovides the structural support of the tissue and significantly determines its characteristic shape and dimensions. It is known that collagen content in the dermis continuously diminishes with progressing age [1], and that it can be additionally reduced as a result of photodamage or of the repeated initiation of remodelling processes $[2,3]$.

The hypothesis that the application of modalities such as radio-frequency current, ultrasound, light sources and so on can locally improve collagen content in the dermis, consequently demonstrating some effects of skin tightening and skin rejuvenation, appears to be reasonable. Indeed, the effect of neocollagenesis is generally considered to be long-term compared to other possible effects

${ }^{*}$ Conflict of interests: Dr. I. Kruglikov is the managing partner of Wellcomet $\mathrm{GmbH}$. such as, for example, hyaluronan and, consequently, water accumulation in a treated area. Such a long-term effect is needed to give the theoretical background to the treatment methods that claim an improvement in skin condition weeks and months after their application. This natural assumption contradicts, however, modern knowledge about the connective tissue structure and especially insight into its turnover, thus making the proclaimed results sometimes controversial and even theoretically impossible.

\section{Some Theoretical Problems in Dermal Aesthetic Treatments}

Every treatment modality that claims that the modification of dermal collagen content is the main effect of its application comes up against at least three problems.

The first one is that the upregulation of multiple stages of collagen production is not necessarily an indication of increased content of mature collagen in the skin. While procollagen can be easily overexpressed after the application of different physical modalities, it can also be easily degraded, which results in a very slow net remodelling of mature collagen.

The second problem is connected with the type of induced collagenesis-physiological (with a normal structure of collagen network) or pathological (scar-like, with typical hyalinised collagen regions). Strengthening of the natural structure of the collagen network in ECM would be the ideal solution, guaranteeing that physiological processes are retained, but this structure is not necessarily in equilibrium and can be reduced relatively rapidly. Pathological neocollagenesis (fibrosis) is connected to the application of higher intensities and results in the 
production of scar-like, intra-tissue structures. These structures can significantly improve the skin rigidity; they are, however, pathologic in their nature. The areas with hyalinised collagen, which is the typical hallmark of keloid scars, will not be refilled with fibroblasts, making later remodelling in this area difficult or even impossible.

The third problem is the dynamic nature of the ECM remodelling, with different characteristic times of the dynamic processes involved. Especially important here is the very slow remodelling dynamic of mature collagen that cannot be significantly modified under quasi-physiological conditions, thus almost elevating the "wounding" of ECM to the necessary condition of effective noninvasive treatment.

To assess the relative importance of procollagen and mature collagen reactions in aesthetic applications, the turnover rates and the relative weighting of these two collagen subpopulations in the total turnover of dermal collagen must be taken into account.

\section{Turnover Rates of Procollagen and Mature Collagen in the Dermis}

Procollagen turnover in the skin is a relatively rapid process with a fractional synthesis rate of about $0.076 \% / \mathrm{h}$ [4]. Supposing there are no degradation processes, the half-time of procollagen renewal can be estimated to be approximately 28 days. In a real situation, a large proportion of newly-produced procollagen will be degraded. The processes of both procollagen synthesis and degradation are strongly dependent on circumference conditions. This makes procollagen very sensitive to different physical factors and, thus, a good candidate for manipulation through the application of different physical modalities.

Contrarily, mature collagen has a very long half-life time, estimated to be approximately 15 years [5]. Such a low turnover rate is mainly connected with its high resistance to proteolytic fragmentation and its relatively high thermic stability. This means that under physiological conditions, mature collagen will only be replaced very slowly through newly-assembled collagen coming from de novo-produced procollagen molecules. Some of the newly-produced, mature collagen will be cleaved by matrix metalloproteinases (MMPs). These processes can be further accelerated by applying different physical factors; for instance, local heating. This rapidly-degraded proportion of neocollagen is not negligible and has been assessed to be $30 \%-50 \%$ in adult rats [6].

Despite the fact that turnover rates of procollagen and mature collagen can vary significantly from one subject to another, these values can be used to estimate the ratio of procollagen to mature collagen content in the dermis. Assuming we can ignore the spontaneous change of the total collagen mass in the dermis under physiological conditions, which is, surely, a reasonable assumption, using the information above we can estimate this ratio to be $0.5 \%$. This value must be considered the upper limit of the estimate, since no procollagen degradation has been taken into account. The order of magnitude of this value is in good agreement with some experimental results, for example, the amount of procollagen in mature, rat skin is about $0.5 \mathrm{mg} / \mathrm{g}$ of the skin mass [7], whereas the total collagen content is about $120 \mathrm{mg} / \mathrm{g}$ [8].

The overall reaction of an ECM to a physical impact can be subdivided into at least three different phases.

\subsection{Activation of Procollagen mRNA Production}

This part of collagen turnover is most sensitive to different physical impacts (e.g. to mechanical forces or heating) and can show a reaction that is sometimes in orders of magnitude higher than the baseline production. This is the "non-specific" reaction, which can be seen after the majority of treatments. Procollagen mRNA production has been measured in different studies and sometimes even used as an indication of neocollagenesis.

\subsection{Production of Procollagen Proteins}

The net amount of procollagen I protein in the dermis is much lower than the procollagen mRNA activation, which is generally connected with its significant degradation. This protein has a high turnover rate and can, in theory, be responsible for some treatment results observed in aesthetic medicine after non-invasive treatments. Its mechanical properties are, however, much weaker compared to mature collagen, and its weighting in the total content of dermal collagen is so low (less than $0.5 \%$ ) that even significant overexpression of procollagen I protein cannot really be seen in the modification of the skin relief either immediately or after some reasonable time post-treatment.

\subsection{Production and Restitution of Mature Collagen Network}

The mature fibrillar collagen network with its rigid structure and high content in the dermis is the only reasonable candidate from the whole collagen pool to be considered responsible for the change in the skin's appearance after non-invasive treatments. Yet, its involvement in these processes is very significantly restricted because of the very low physiological turnover rate of the mature collagen. This collagen network cannot be directly modified under quasi-physiological conditions due to its high proteolytic resistance and can be only slowly replaced through the production of procollagen and degradation of old and damaged collagen structures. Only 
the accumulation of the damaged collagen structures, which can be neither repaired nor eliminated, can be responsible for the reduced activity of fibroblasts and, thus, finally for skin ageing [3].

\section{Quasi-Physiological and Pathological Conditions}

Mechanical properties as well as, to some extent, the optical appearance of the skin are dependent on the concentration of mature fibrillar collagen (especially on $\mathrm{Col}$ I, which makes up more than $90 \%$ of the skin's dry weight), organised into the large enzymatically crosslinked and proteolytically resistant fibres. The mature collagen can, however, only be produced from procollagen, which takes the form of a soluble triple helix and is assembled inside of fibroblasts and is then either degraded intracellularly or secreted from fibroblasts into the ECM. Procollagen molecules in the ECM can lose their N- and C-terminal pro-peptides and then spontaneously assemble into large, non-soluble, mature collagen fibres. The latter can be also enzymatically degraded by specific MMPs; this degradation is, however, extremely slow and cannot significantly influence the short- and mid-term results of aesthetic treatments, as long as the stimulation, and not the damage processes, takes place in the dermis.

All dynamic components - production of procollagen, maturing of collagen, destruction of procollagen and mature collagen, and regulation of the pool of active fibroblasts capable of producing the components of ECM - can be modified in different ways through various aesthetic modalities. The activation of procollagen mRNA and protein production is already possible under quasiphysiological conditions and can be observed after different, non-damaging aesthetic treatments, whereas the overexpression of procollagen protein is generally much less pronounced than that of procollagen mRNA. The production of specific MMPs can also take place as a result of a physiological reaction. At the same time, this production is tightly connected with local collagen content through feedback mechanism, thus making it possible to cleave, short-term, a significant part of de novoproduced collagen. These processes can increase the turnover of ECM, but the feedback mechanism between the synthesis and the degradation of collagen will restrict the uncontrolled collagen accumulation preventing fibrosis.

The significant migration and production of new fibroblasts do not normally take place under quasi-physiological conditions. These phenomena can, however, be of significant importance in severe tissue damage, for example, in the healing of wounds or in skin resurfacing procedures, where quick and strong repair is required. This makes evident the significant difference between the processes involved in skin rejuvenation and in wound healing, which are often considered to be very similar.

The following example demonstrates that different physiological mechanisms can lead to a similar treatment endpoint through the dynamic balancing of the remodelling processes. It has been shown [9] that the same subjects can have a very different relationship with the synthesis of procollagen and the expression of MMPs in different body regions. The areas with low sun exposure (chronological skin ageing) in older people demonstrate significantly decreased production of procollagen I mRNA and the increased expression of MMPs, when compared to the same body areas in younger people. In the areas of strong sun exposure (photoageing), the procollagen I mRNA production was, however, on average 2.9 times higher and the MMPs were more strongly overexpressed than in the areas with pure chronological ageing in the same subjects. Both processes (chronological and photoinduced ageing) led to the same endpoint - total collagen deficiency. However, the net amount of procollagen I protein in the dermis of photoaged skin, which demonstrated a higher synthesis of procollagen I mRNA, was much lower. This apparently paradoxical result, which is mainly connected with the different expression of MMPs, gave the authors possibility to speculate about the potentially different pathophysiologies of chronological and photo-induced skin ageing.

Under quasi-physiological conditions, the connective tissue remodelling is in a dynamic balance between the production and destruction of its matrix components. Each instance of overproduction of collagen or other ECM components will, in the short-term, initiate the feedback mechanism, causing the stimulation of their enzymatic degradation and thus compensating the large part of the stimulatory effect. For example, the $532 \mathrm{~nm}$ KPT and the $1064 \mathrm{~nm}$ Q-switched Nd:YAG laser at intensities of $1.5 \mathrm{~J} / \mathrm{cm}^{2}$ can increase the expression of procollagens I and III mRNAs and simultaneously decrease the expression of MMP-1 and MMP-2 [10], consequently suppressing the cleavage of collagen. At the same time, the ablative application of $\mathrm{CO}_{2}$ laser could significantly increase the induction of procollagen I (by up to 7.5 times the original value, 21 days after the procedure) and III mRNAs and MMP-1 mRNA (by up to 40,000 times, seven days after the procedure), which also correlates with the dynamical change of MMP-1 protein levels [11]. Whereas the net amount of procollagen I protein was also increased, up to six months after $\mathrm{CO}_{2}$ laser treatment, its increase was much lower than that of procollagen I mRNA, clearly demonstrating the balancing effect. The qualitative difference between the dynamic processes in the dermis after the application of these two treatment modalities may be connected with much stronger amplitudes of dynamic changes after applying $\mathrm{CO}_{2}$ laser. It has 
even been assumed that different laser applications can cause different mechanisms of collagen destruction [12].

Let us estimate how realistic the change in the skin's appearance through quasi-physiological remodelling of the mature collagen network is. We will assume the physiological half-life time of the mature collagen in the dermis to be 15 years. The increase of the procollagen I protein in the dermis will be taken to be 2.4 , as, for example, the maximum value measured after one skin rejuvenation treatment with photodynamic therapy recorded seven days after the procedure [13]. Assuming the whole procollagen I protein will be utilised to replace the mature protein and that the procollagen upregulation is constant during the whole time after the treatment (which is surely wrong and will cause us to significantly overestimate the results), the proportion of the mature collagen network that will be replaced during the first seven days after the treatment can be calculated to be approximately $0.15 \%$ (the upper-limit of the estimation). Realistically, this value has to be reduced at least twice. It is absolutely unrealistic that such a modification can significantly improve the skin appearance. Even assuming the average procollagen I protein upregulation to be $2.0 \%$ during the first 30 days, only $0.55 \%$ of the local network will be remodelled.

The change of procollagen I mRNA of 2.4- to 1.7-fold, correspondingly two and seven days after RF treatment of the skin [14], also cannot be responsible for any improvement in the skin's appearance, since the overexpression of the procollagen I protein in this case has to be even smaller than in the study of [13].

The treatment results and their interpretation can vary greatly if the treatments under quasi-physiological conditions are regularly repeated, supporting, in the long-term, a higher level of collagen turnover, and if the skin assessment is done after a long treatment time. Such a case was investigated by [15] for the application of tretinoin. An $80 \%$ increase of dermal procollagen I was registered after approximately 12 months of daily treatments, which can, thus, cause the remodelling of about $6 \%$ of the fibrillar collagen network.

It is also possible to obtain stronger treatment results through direct collagen damage, or "wounding", and its possible degradation through MMPs.

\section{Collagen Damage and Degradation}

The degradation of collagen is a very sophisticated process, which has both extracellular and intracellular pathways. Extracellular degradation is mediated by some MMPs and is specific to rapidly growing or damaged tissues. Under physiological conditions, collagen degradation is primarily connected to an internal pathway, which is often named collagen phagocytosis. This process is independent of MMP-1 activity, but can be significantly modulated by MT1-MMP [16].

Significant reduction in collagen phagocytosis has been observed in fibroblasts growing on a partly denatured collagen matrix $[17,18]$. Chronic denaturing of the collagen matrix by MMPs can lead to an accumulation of fragmented collagen, which cannot be completely removed from the matrix because of its residual crosslinking. If this fragmentation concerns a significant part of the matrix (according to estimations done in [2] this can be about $30 \%-40 \%$ of all collagen molecules), that which is normally homogeneous in the large scale ECM structure will appear clumpy. Fibroblasts will have not had the possibility to properly attach to the matrix, which would normally be the necessary condition for their active collagen production. Improvement of the matrix structure through the elimination of fragments would surely also increase the number of active fibroblasts, thus indirectly increasing the collagen turnover. The physiological elimination of fragmented collagen is, however, limited by the low characteristic turnover rate of the collagen network. This restricts the possibility of treatment methods used to improve the skin structure in short- or mid-term intervals under quasi-physiological conditions. One alternative possibility would be the strong, additional damage of the collagen network with the production of scar-like structures.

At the same time, both collagen phagocytosis and defragmentation of the matrix cannot dynamically improve the skin appearance during the observation times claimed in most non-invasive or minimal invasive aesthetic modalities, because of the low characteristic turnover rate of mature collagen.

\section{Conclusion}

There are well-established correlations between the improvement in the skin's appearance after the application of different non-invasive or minimal invasive aesthetic treatment modalities, and dermal neocollagenesis. Such a correlation has been demonstrated using laser and IPL, LED, ultrasound, radio frequency and other applications. This correlation is often interpreted as evidence that the visible skin modifications are caused by newly-produced collagen. However, the very slow dynamics of the mature collagen remodelling in the ECM of dermis, with a half-life time of 15 years, renders every observable upregulation of the collagen production insufficient to replace a significant part of the matrix during the short time in which it is claimed skin improvement takes place. Consequently, neocollagenesis correlates temporally with short- and mid-term skin improvement but cannot be its 
main reason, which is often assumed in clinical studies.

\section{REFERENCES}

[1] J. Varani, R. L. Warner, M. Gharaee-Kermani, S. H. Phan, S. Kang, J. H. Chung, Z. Q. Wang, S. C. Datta, G. J. Fisher and J. J. Voorhees, "Vitamin A Antagonizes Decreased Cell Growth and Elevated Collagen-Degrading Matrix Metalloproteinases and Stimulates Collagen Accumulation in Naturally Aged Human Skin," Journal of Investigative Dermatology, Vol. 114, No. 3, 2000, pp. 480486. doi:10.1046/j.1523-1747.2000.00902.x

[2] S. E. G. Fligiel, J. Varani, S. C. Datta, S. Kang, G. J. Fisher and J. J. Voorhees, "Collagen Degradation in Aged/ Photodamaged Skin in Vivo and after Exposure to Matrix Metalloproteinase-1 in Vitro," Journal of Investigative Dermatology, Vol. 120, No. 5, 2003, pp. 842-848. doi:10.1046/j.1523-1747.2003.12148.x

[3] G. J. Fisher, J. Varani and J. J. Voorhees, "Looking Older. Fibroblast Collapse and Therapeutic Applications," Archive of Dermatology, Vol. 144, No. 5, 2008, pp. 666-672.

[4] W. A. El-Harake, M. A. Furman, B. Cook, K. S. Nair, J. Kukowski and I. G. Brodsky, "Measurement of Dermal Collagen Synthesis Rate in Vivo in Humans," American Journal of Physiology, Vol. 274, No. 4, 1998, pp. E586E591.

[5] N. Verzijl, J. DeGroot, S. R. Thorpe, R. A. Bank, J. N. Shaw, T. J. Lyons, Bijlsma, F. P. Lafeber, J. W. Baynes and J. M. TeKoppele, "Effect of Collagen Turnover on the Accumulation of Advanced Glycation End Products," Journal of Biological Chemistry, Vol. 275, No. 15, 2000, pp. 39027-39031. doi:10.1074/jbc.M006700200

[6] P. R. Mays, R. J. McNulty, J. S. Campa and G. J. Laurent, "Age-Related Changes in Collagen Synthesis and Degradation in Rat Tissues: Importance of Degradation of Newly Synthesized Collagen in Regulating Collagen Production," Biochemistry Journal, Vol. 276, 1991, pp. 307-313.

[7] Z. O. Wirtschaftler and A. S. Bentley, "The Influence of Age and Growth Rate on the Extractable Collagen of Skin of Normal Rats," Laboratory Investigations, Vol. 11, 1962, pp. 316-320.

[8] J. C. Waterlow and J. M. L. Stephen, "Adaptation of the Rat to a Low-Protein Diet: The Effect of a Reduced Protein Intake on the Pattern of Incorporation of L- $\left[{ }^{14} \mathrm{C}\right] \mathrm{Ly}-$ sine," British Journal of Nutrition, Vol. 20, No. 3, 1966, pp. 461-484. doi:10.1079/BJN19660047

[9] J. H. Chung, J. Y. Seo, H. R. Choi, M. K. Lee, C. S. Youn, G. Rhie, K. H. Cho, K. H. Kim, K. C. Park and H. C. Eun, "Modulation of Skin Collagen Metabolism in Aged and Photoaged Human Skin in Vivo," Journal of Investigative Dermatology, Vol. 117, No. 5, 2001, pp. 1218-1224. doi:10.1046/j.0022-202x.2001.01544.x

[10] Y. Dang, X. Ye, Y. Weng, Z. Tong and Q. Ren, "Effects of the 532-nm and 1064-nm Q-Switched Nd:YAG Lasers on Collagen Turnover of Cultured Human Skin Fibroblasts: A Comparative Study," Lasers in Medical Sciences, Vol. 25, No. 5, 2010, pp. 719-726. doi:10.1007/s10103-009-0657-4

[11] J. S. Orringer, S. Kang, T. M. Johnson, D. J. Karimipour, T. Hamilton, C. Hammerberg, J. J. Voorhees and G. J. Fischer, "Connective Tissue Remodeling Induced by Carbon Dioxide Laser Resurfacing of Photodamaged Human Skin," Archives of Dermatology, Vol. 140, No. 11, 2004, pp. 1326-1332. doi:10.1001/archderm.140.11.1326

[12] C. Spock, A. I. Metelitsa, J. Kaufman and J. B. Green, "Lasers and Light Sources to Activate Fibroblasts," Cosmetical Dermatology, Vol. 25, No. 1, 2012, pp. 27-33.

[13] J. S. Orringer, C. Hammerberg, T. Hamilton, T. M. Johnson, S. Kang, D. L. Sachs, G. J. Fisher and J. J. Voorhees, "Molecular Effects of Photodynamic Therapy for Photoaging," Archives of Dermatology, Vol. 144, No. 10, 2008, pp. 1296-1302. doi:10.1001/archderm.144.10.1296

[14] B. D. Zelickson, D. Kist, E. Bernstein, D. B. Brown, S. Ksenzenko, J. Burns, S. Kilmer, D. Mehregan and K. Pope, "Histological and Ultrastructural Evaluation of the Effects of a Radiofrequency-Based Nonablative Dermal Remodeling Device. A Pilot Study," Archives of Dermatology, Vol. 140, No. 2, 2004, pp. 204-209. doi:10.1001/archderm.140.2.204

[15] C. E. M. Griffiths, A. N. Russman, G. Majmudar, R. S. Singer, T. A. Hamilton and J. J. Voorhees, "Restoration of Collagen Formation in Photodamaged Human Skin by Tretinoin (Retinoic Acid)," New England Journal of Medicine, Vol. 329, No. 8, 1993, pp. 530-535. doi:10.1056/NEJM199308193290803

[16] H. Lee, C. M. Overall, C. A. McCulloch and J. Sodek, “A Critical Role for the Membrane-Type 1 Matrix Metalloproteinase in Collagen Phagocytosis," Molecular Biology of Cell, Vol. 17, No. 11, 2006, pp. 4812-4826. doi:10.1091/mbc.E06-06-0486

[17] J. Varani, D. Spearman, P. Perone, S. E. G. Fligiel, S. C. Datta, Z. Q. Wang, Y. Shao, S. Kang, G. J. Fisher and J. J. Voorhees, "Inhibition of Type I Procollagen Synthesis by Damaged Collagen in Photoaged Skin and by CollagenaseDegraded Collagen in Vitro," American Journal of Pathology, Vol. 158, No. 3, 2001, pp. 931-942. doi:10.1016/S0002-9440(10)64040-0

[18] L. C. Abraham, J. F. Dice, K. Lee and D. L. Kaplan, "Phagocytosis and Remodeling of Collagen Matrices," Experimental Cell Research, Vol. 313, No. 5, 2007, pp. 10451055. doi:10.1016/j.yexcr.2006.12.019 